\title{
Análisis de parámetros histopatológicos y clínicos en pacientes caninas con neoplasias mamarias tratadas con tamoxifeno
}

\author{
Merlo, W.A.; Rosciani, A.S.; Insfrán, R.M.; López, J.E.; Macció, O.A. \\ Servicio de Diagnóstico Histopatológico y Citológico, Hospital de Clínicas, Facultad de Ciencias Veterinarias, \\ UNNE. Sargento Cabral 2139, Corrientes (3400), Argentina. Tel/fax.:03783-425753, Int. 175. \\ E-mail: histopat@vet.unne.edu.ar
}

\begin{abstract}
Resumen
Merlo, W.A.; Rosciani, A.S.; Insfrán, R.M.; López, J.E.; Macció, O.A.: Análisis de parámetros histopatológicos y clínicos en pacientes caninas con neoplasias mamarias tratadas con tamoxifeno. Rev. vet. 21: 1, 34-37, 2010. Esta comunicación tiene como objetivo presentar los resultados obtenidos de la evaluación de diferentes parámetros en doce perras con neoplasias mamarias, antes y después del tratamiento con tamoxifeno. Las pacientes se encontraban en estadios clínicos III y IV determinados de acuerdo con el sistema TNM (tumor, nódulo linfático, metástasis) de la Organización Mundial de la Salud. Se efectuaron punciones con aguja fina para citodiagnóstico, biopsias pre-tratamiento y extirpación quirúrgica de las mamas afectadas post-tratamiento. El estudio histopatológico de estas piezas permitió su clasificación, determinación del grado tumoral y evaluación de parámetros histológicos como presencia de necrosis, invasión vascular e infiltración linfoplasmocitaria. El citodiagnóstico fue coincidente con los diagnósticos histopatológicos de las piezas quirúrgicas post-tratamiento en el $50 \%$ de los casos. El análisis histopatológico de las biopsias mostró diferencias en la clasificación, graduación histológica de malignidad y en la determinación de invasión vascular, infiltrado linfoplasmocitario y necrosis, con las piezas quirúrgicas post-tratamiento. Se considera que estas diferencias se deberían al pequeño tamaño de las biopsias pre-quirúrgicas, que no reflejarían la típica heterogeneidad de estos tumores. No se verificaron cambios importantes atribuibles a la acción del tamoxifeno, cuyo lapso de administración fue de corta duración y aplicado a pacientes con estadios avanzados de la enfermedad.
\end{abstract}

Palabras clave: perra, neoplasia mamaria, tamoxifeno, parámetros diagnósticos.

\begin{abstract}
Merlo, W.A.; Rosciani, A.S.; Insfrán, R.M.; López, J.E.; Macció, O.A.: Analysis of histopathological and clinical parameters in canine patients with mammary neoplasms treated with tamoxifen. Rev. vet. 21: 1, 34-37, 2010. The purpose of this work is to present the results from the evaluation of different parameters in twelve female dogs with mammary tumors before and after treatment with tamoxifen. Animals were in clinical stages III and IV according to the World Health Organization TNM system (tumor, node, metastasis). Samples obtained using fine needle aspiration cytodiagnosis and pre-treatment tumor biopsies were analyzed. Mammary gland extirpations were performed after tamoxifen treatment. Histopathology was performed to classify the neoplasms, as well as to determine histological malignancy grade and to evaluate other parameters such as presence of necrosis, vascular invasion, and lymphoplasmacytic infiltration. The cytodiagnosis was in concordance with the histological classification of surgical specimens in $50 \%$ of the cases. The histological classification, the histological grade of malignancy determination, and the assessment of vascular invasion, lymphoplasmacytic infiltration, and necrosis showed many differences between pre-treatment biopsies and post-treatment surgical samples. These differences are considered as a consequence of the small size of the biopsies that would not reflect the particular heterogeneity of the tumors. Furthermore, these differences do not allow to confirm neither reject tamoxifen pharmacological effectiveness, which period of administration was short and implemented in patients in advanced clinical stages.
\end{abstract}

Key words: bitch, mammary neoplasm, tamoxifen, diagnostic factors. 


\section{INTRODUCCIÓN}

Los tumores mamarios caninos son neoplasias heterogéneas tanto en sus aspectos morfológicos como en su comportamiento clínico. Esta situación, los convierte en un importante objeto de análisis, en búsqueda de diferentes parámetros clínicos e histopatológicos con valor pronóstico y/o terapéutico. Debido a que la ablación quirúrgica de los tumores mamarios malignos no es curativa, se ha investigado la eficacia de terapias adyuvantes. Aunque no existen datos hasta el momento para confirmar los efectos beneficiosos de tales medidas en las perras y gatas, la quimioterapia a menudo se utiliza como adyuvante del tratamiento quirúrgico de los tumores mamarios ${ }^{7}$.

El tamoxifeno es el quimioterápico más exitoso en el manejo del cáncer mamario en medicina huma$\mathrm{na}^{8}$. Actúa interfiriendo con la actividad del estrógeno, uniéndose en forma competitiva a sus receptores y evitando el crecimiento de células cancerosas en la glándula mamaria promovido por la hormona. En los caninos, a diferencia de lo que ocurre en pacientes humanos, el tamoxifeno tiene efectos estrogénicos ${ }^{7}$ y su uso veterinario ha brindado resultados contradictorios $3,6,7,9,10$. Se mencionan efectos colaterales tales como piómetra, hiperplasia vaginal y/o fiebre, por lo que se imponen nuevos estudios a fin de ajustar el régimen de administración ${ }^{6}$. La dosis utilizada en caninos es de 0,4 a 0,8 $\mathrm{mg} / \mathrm{kg} /$ día por vía oral durante 4 a 8 semanas ${ }^{10}$.

Las neoplasias mamarias caninas se han agrupado de acuerdo a criterios histogénico, morfológico descriptivo y pronóstico, resultando en clasificaciones innecesariamente complejas y de difícil aplicación. La clasificación actual de la Organización Mundial de la Salud se ha simplificado y relaciona los diferentes tipos histológicos con el grado de malignidad ${ }^{4}$.

El examen histopatológico constituye el método diagnóstico más confiable, ya que permite además de la tipificación y graduación, la valoración de factores como invasión vascular, cutánea y de tejidos blandos ${ }^{4}$. La escasa diferenciación (histológica y nuclear) y la invasión vascular y linfática se asocian de manera constante en los casos de peor pronóstico, aunque existen variaciones entre observadores en su valoración ${ }^{2}$. Numerosos trabajos de oncología humana aseveran que la invasión vascular marca un punto crucial para el pronóstico. La presencia de grandes zonas de necrosis dentro de la masa tumoral es un signo que empeora el pronóstico ${ }^{1}$.

Tabla 1. Estadificación de los tumores mamarios en perras.

\begin{tabular}{lccc}
\hline estadio & tumor primario & nódulo linfát. reg. & metástasis dist. \\
\hline 1 & $\mathrm{~T} 1$ & $\mathrm{~N} 0$ & $\mathrm{M} 0$ \\
2 & $\mathrm{~T} 2$ & $\mathrm{~N} 0$ & $\mathrm{M} 0$ \\
3 & $\mathrm{~T} 3$ & $\mathrm{~N} 0$ & $\mathrm{M} 0$ \\
4 & cualquier T & $\mathrm{N} 1$ & $\mathrm{M} 0$ \\
5 & cualquier T & cualquier N & $\mathrm{M} 1$ \\
\hline
\end{tabular}

El informe histopatológico debe incluir siempre el grado de infiltración y el grado histológico de malignidad. El grado de malignidad histológica usualmente se expresa en una escala de 1 a 3 grados, donde el grado 3 tiene los peores pronósticos ${ }^{4,5}$. En esta comunicación se presentan algunos hallazgos obtenidos de pacientes caninas con neoplasias mamarias tratadas con tamoxifeno.

\section{MATERIAL Y MÉTODOS}

Se evaluaron doce hembras caninas con neoplasias mamarias que asistieron a la consulta al Hospital de Clínicas de la Facultad de Ciencias Veterinarias de la UNNE (Corrientes, Argentina). En los casos en que dichas pacientes presentaron más de un tumor mamario, se seleccionó el de mayor tamaño para su evaluación. Se realizó punción con aguja fina de los tumores para citodiagnóstico, empleando agujas descartables 40 × 8 . Luego se efectuaron biopsias tumorales (en cuña), pretratamiento con tamoxifeno y posteriormente al mismo, mastectomía. Las pacientes recibieron el quimioterápico a razón de $0,4 \mathrm{mg} / \mathrm{kg} /$ día por vía oral durante diez días.

Con los datos obtenidos de la exploración clínica, el estudio citológico de los nódulos linfáticos hipertróficos, las radiografías de tórax y las ecografías de abdomen se realizó la estadificación clínica de las pacientes, basada en la clasificación TNM (OMS) ${ }^{9}$. Este sistema tiene en cuenta tamaño del tumor (T), compromiso ganglionar $(\mathrm{N})$ y posibles metástasis $(\mathrm{M})$, acorde a las siguientes claves:

- T-Tumor primario: T1: $<3 \mathrm{~cm}$ de diámetro, T2: de 3 a $5 \mathrm{~cm}$, T3: $>5 \mathrm{~cm}$.

- N-Ganglio regional: N0: sin metástasis, N1: metástasis.

- M - Metástasis distante: M0: sin metástasis, M1: metástasis.

En la Tabla 1 se expone el sistema de estadios clínicos de la Organización Mundial de la Salud modificado por Owen (1980) para estadificación de los tumores mamarios caninos ${ }^{9}$.

El estudio histopatológico de las neoplasias permitió su diagnóstico teniendo en cuenta la clasificación histológica de los tumores mamarios del perro y el gato ${ }^{4}$, la determinación del grado de malignidad tumoral ${ }^{5}$ y la evaluación de parámetros histológicos como la presencia de necrosis, invasión vascular e infiltración linfoplasmocitaria. El grado de malignidad tumoral se basa en el sistema de graduación histológica de los carcinomas mamarios caninos y felinos propuesto por Misdorp (2002) ${ }^{5}$, que cuantifica las siguientes características:

(a) formación de túbulos (1 punto: formación tubular bien marcada, 2 puntos: mediano desarrollo tubular, 3 puntos: formación tubular escasa o nula).

(b) pleomorfismo nuclear (1 punto: núcleos uniformes o regulares en tamaño, forma y coloración, 2 puntos: moderada variación en el tamaño y forma nuclear 
y ocasionales nucléolos, 3 puntos: marcada variación en el tamaño nuclear, núcleos bizarros y frecuentes nucléolos prominentes).

(c) índice mitótico, mitosis e hipercromasia (1 punto: si solo hay una figura mitótica por campo de gran aumento, 2 puntos: dos o tres figuras mitóticas por campo, 3 puntos: si el número de figuras mitóticas por campo es más alto).

Grado final de malignidad: se obtiene por la suma de los tres parámetros observados. Grado I (carcinoma bien diferenciado: 3 a 5 puntos), grado II (carcinoma moderadamente diferenciado: 6 a 7 puntos) y grado III (carcinoma poco diferenciado: 8 a 9 puntos).

\section{RESULTADOS}

Estadios clínicos. Al momento de la primer consulta, las pacientes caninas estudiadas $(\mathrm{n}=12)$ se encontraban en estadio clínico III $(\mathrm{n}=6)$ y en estadio $I V$ $(\mathrm{n}=6)$, situación que se mantuvo luego del tratamiento, confirmado por el estudio histopatológico de los nódulos linfáticos regionales extirpados post-tratamiento (Figuras 1 y 2).

Análisis citológicos. En seis casos los tumores fueron diagnosticados citológicamente como compatibles con carcinomas en tumor mixto y dichos diagnósticos coincidieron con los resultados histopatológicos de esas neoplasias post-tratamiento. Los seis casos restantes tuvieron un diagnóstico orientativo ya que sólo se informó la presencia de células carcinomatosas.

Estudios histopatológicos. El análisis de las biopsias tumorales determinó los siguientes diagnósticos: carcinomas simples $(\mathrm{n}=6)$, carcinomas en tumor mixto $(\mathrm{n}=3)$ y carcinomas complejos $(\mathrm{n}=3)$. Dichos diagnósticos no concordaron plenamente con los realizados sobre las piezas quirúrgicas post-tratamiento. En efecto, el estudio histopatológico de estas últimas determinó que sólo tres de esas neoplasias fueron carcinomas simples y las nueve restantes carcinomas en tumor mixto.

Grados de malignidad tumoral. En las biopsias pre-tratamiento, seis tumores fueron asignados como grado I y tres correspondieron al grado II. No fue po-

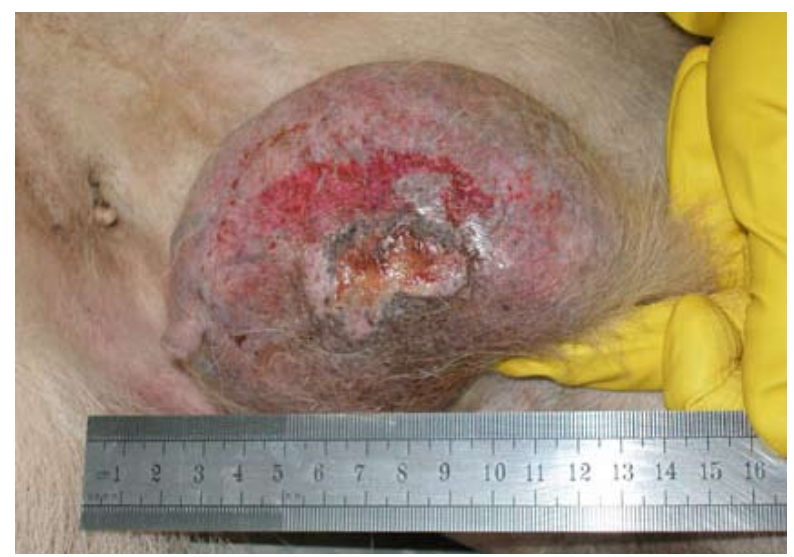

Figura 1. Medición del tumor mamario. TNM. sible efectuar esta graduación en tres ocasiones correspondientes a tumores mixtos debido a la ausencia, en las muestras, de áreas carcinomatosas. El análisis de las piezas quirúrgicas de las neoplasias post-tratamiento mostró que los tumores considerados en las biopsias como de grado I, aquí se manifestaron como grado II, mientras que los de grado II se mantuvieron en la misma graduación luego del tratamiento.

Invasión vascular y linfática fue observada en los cortes de las piezas quirúrgicas post-tratamiento de tres pacientes en estadio clínico $I V$, no así en las biopsias (Figura 3). Necrosis: se observó en general, un aumento en las áreas de necrosis en las mamas post-tamoxifeno. Infiltrado linfoplasmocitario: se halló sólo en las piezas post-tratamiento de todos los tumores analizados. Tres de las pacientes presentaron complicaciones como hiperplasia endometrial y metritis, considerados efectos colaterales de tipo estrogénico.

\section{DISCUSIÓN}

La aplicación del sistema de estadios clínicos para los tumores mamarios caninos ${ }^{9}$ resultó práctica al momento de estadificar nuestras pacientes. El examen histopatológico de las piezas quirúrgicas se manifes-

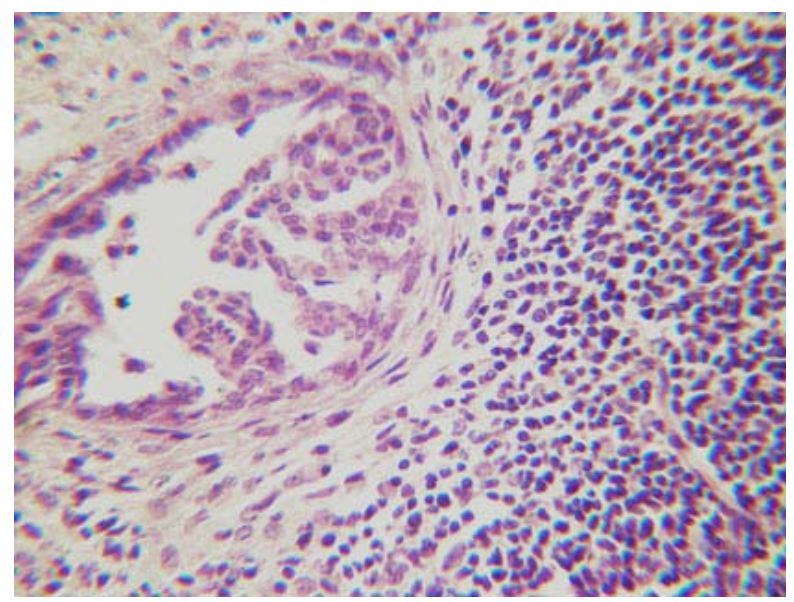

Figura 2. Foco de metástasis de carcinoma mamario en nódulo linfático (HyE, 400x).

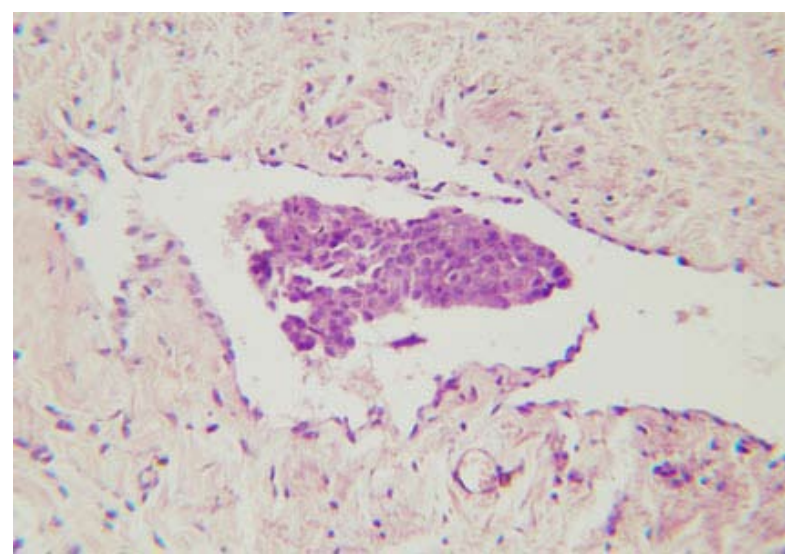

Figura 3. Émbolo neoplásico en vaso linfático (HyE, 400x). 
tó como el método diagnóstico de elección, como lo mencionan otros autores, por permitir una evaluación integral del tejido neoplásico ${ }^{4}$. Las diferencias encontradas en la clasificación de los tumores por medio de las distintas técnicas de muestreo, evidenciaron una mayor correlación entre el citodiagnóstico y el estudio histopatológico de las piezas quirúrgicas.

Las biopsias resultaron de limitada utilidad para el diagnóstico de los tumores mamarios ya que no reflejaron la particular heterogeneidad de algunas variedades histopatológicas de estas neoplasias. Probablemente debido a su escasa extensión, hubo dificultades en la determinación del grado de malignidad tumoral y no permitieron evidenciar la presencia de invasión vascular, ni de infiltrado linfoplasmocitario. El hallazgo de invasión vascular en las pacientes de estadio clínico más elevado (IV), se encuentra dentro de lo esperado al considerar a este fenómeno como factor de mal pronóstico ${ }^{2}$.

En cuanto a la acción del tamoxifeno, no fue posible en estos casos y con la dosificación final utilizada, observar cambios llamativos en el tejido tumoral estudiado entre las biopsias pre-tratamiento y las piezas quirúrgicas post-tratamiento. Consideramos importante señalar que la mayoría de las pacientes llegaron en estadios avanzados de la enfermedad, situación que resultaría difícil revertir con un tratamiento corto. Por otro lado, se observa que lo sugerido por numerosos autores con respecto a las complicaciones por los efectos estrogénicos de esta droga se evidenciaron en algunas pacientes, a pesar de haber pretendido evitarlas con una administración de menor duración.

\section{REFERENCIAS}

1. Bostock DE. 1975. The prognosis following the surgical excision of canine mammary neoplasms. Eur J Cancer 11 : 389-396.

2. Dickson RB, Lippman ME. 2000. Cáncer de mama. En: Cáncer. Principios y práctica de oncología (De Vita VT, Hellman S, Rosenberg SA, Ed.), Panamericana, Madrid, p.1541-1616.

3. Kitchell GN. 1995. Mammary tumors. In: Current Veterinary Therapy. XII. Small Animal Practice (Kirk RW Ed.), Saunders, Philadelphia, p. 1098-1103.

4. Misdorp W, Else RW, Hellmen E, Lipscomb TP. 1999. Histological classification of mammary tumors of the dog and the cat. Second series, Volume VII. Published by the Armed Forces Institute of Pathology with WHO, Washington, $58 \mathrm{p}$.

5. Misdorp W. 2002. Tumors of the mammary gland. In: Tumors in domestic animals (Meuten DJ Ed.), $4^{\text {th }}$ ed., Blackwell Publishing, Iowa, p. 575-606.

6. Morris JS, Dobson JM, Bostock DE. 1993. Use of tamoxifen in the control of canine mammary neoplasia. Vet Rec 133: 539-542.

7. Nelson RW, Couto CG. 2005. Medicina interna de animales pequeños, Intermédica, Buenos Aires, p. 930-934.

8. Osborne CK, Jarman M, McCague R, Coronado EB, Hilsenbek SG, Wakeling AE. 1994. The importance of tamoxifen metabolism in tamoxifen-stimulated breast tumor growth. Cancer Chemother Pharmacol 34: 89-95.

9. Owen LN. 1980. TNM classification of tumours in domestic animals, Publ. World Health Organization, Geneva, p. 16-20.

10. Tanaka N. 2003. Tumor de mama: Qual a melhor conduta? Boletín Informativo Anclivepa, San Pablo, 29: 6-7. 